\title{
Concurrent Osteosarcoma Theranostic Strategy Using Contrast-Enhanced Ultrasound and Drug-Loaded Bubbles
}

\author{
Tai-Tzung Kuo ${ }^{1,2}$, Chung-Hsin Wang ${ }^{1}$, Jir-You Wang ${ }^{3,4,5}$, Hong-Jen Chiou ${ }^{6}$, Ching-Hsiang Fan ${ }^{1}$ \\ and Chih-Kuang Yeh $1,7, *$ (D) \\ 1 Department of Biomedical Engineering and Environmental Sciences, National Tsing Hua University, Hsinchu \\ 30013, Taiwan; 4348@mmh.org.tw (T.-T.K.); chwang@trust-biosonics.com (C.H.W.); s1024@msn.com (C.-H.F.) \\ 4 Department of Orthopaedics, Therapeutical and Research Center of Musculoskeletal Tumor, Taipei Veterans \\ General Hospital, Taipei 11217, Taiwan \\ 5 Institute of Traditional Medicine. National Yang-Ming University, Taipei 11221, Taiwan \\ 6 Department of Radiology, Taipei-Veterans General Hospital, Taipei 11217, Taiwan; hjchiou@vghtpe.gov.tw \\ 7 Institute of Nuclear Engineering and Science, National Tsing Hua University, Hsinchu 30013, Taiwan \\ * Corresponding: ckyeh@mx.nthu.edu.tw; Tel.: +886-3-5715131 (ext. 34240); Fax: +886-3-5718649
}

Received: 1 April 2019; Accepted: 6 May 2019; Published: 8 May 2019

\begin{abstract}
Osteosarcoma (OS) is the most common bone tumor in children and teenagers. The multidrug resistant property of OS produces a major obstacle to chemotherapy, since the effective drug dose cannot be achieved via conventional drug delivery routes without serious systemic cytotoxicity. Microbubbles in conjunction with ultrasound (US) has recently been shown to spatially and temporally permeabilize the cellular membrane, promoting drug penetration into tumors. Here, we investigated whether drug (doxorubicin, DOX)-loaded bubbles (DOX-bubbles) can serve as drug-loaded carriers in combination with US in order to facilitate tumor drug delivery. The proposed bubbles have a high payload capacity (efficiency of $69.4 \pm 9.1 \%$, payload of $1.4 \mathrm{mg} / \mathrm{mL}$ ) for DOX. In vitro data revealed that when used in combination with US (1-MHz), these DOX-bubbles facilitate DOX entering into tumor cells. In tumor-bearing animals, DOX-bubbles + US could provide 3.7-fold suppression of tumor growth compared with the group without insonation $\left(1.8 \pm 0.9 \mathrm{~cm}^{3} \mathrm{vs}\right.$. $8.5 \pm 2.2 \mathrm{~cm}^{3}$ ) because of the acceleration of DOX-induced tumor necrosis. In the meantime, the tumor perfusion and volume can be monitored by DOX-bubbles with contrast-enhanced ultrasound imaging. Our data provide useful information in support of translating the use of theranostic US-responsive bubbles for regulated tumor drug delivery into clinical use.
\end{abstract}

Keywords: microbubbles; contrast-enhanced ultrasound; osteosarcoma; enhanced permeability; drug-loaded; ultrasound contrast agents; therasostics

\section{Introduction}

Osteosarcoma (OS) is the most common kind of bone cancer in children and teenagers [1]. It is characterized by high proclivity for early systemic metastases and local invasion [2]. The current approved clinical treatments for OS include surgery and chemotherapy (such as doxorubicin, DOX) [3,4]. The cure rate in OS patients ranges from 15 to $20 \%$ with surgery alone, but improves to nearly $70 \%$ when the surgery is performed in conjunction with chemotherapy [4]. Despite advances in surgical techniques and neoadjuvant chemotherapy regimens, the failure of cure with OS is still about $30 \%$, which is mainly 
due to the development of multidrug resistance [4,5]. Developing novel chemotherapeutic strategies to overcome multidrug resistance of OS is necessary.

The decrease in intracellular drug efflux caused by P-glycoprotein (P-gp) plays an important role in multidrug resistance in OS therapy. The limited drug accumulation results in failure to respond to DOX [6]. In order to overcome the drug resistance caused by P-gp, numerous approaches have been broadly proposed, including the administration of DOX-carrying lipid-based nanoparticles or combinations of DOX with other chemotherapeutic agents (i.e., cisplatin, methotrexate) $[7,8]$. However, a nanoscale drug delivery system probably affects all organs during circulation, leading to accumulation of the cytotoxic agents in undesired areas; several classes of administrated drug for treating OS can lead to systemic cytotoxic effects.

The use of ultrasound (US) exposure in the presence of microbubbles has been approved in pre-clinical studies as a noninvasive and reversible approach to enhance permeability in the local area, leading to a transient opportunity for facilitating delivery of therapeutic substances to a target site [9-13]. Further, this approach can produce cardio- and nephrotoxicity due to direct exposure to the chemo-drug in blood circulation [14]. The encapsulation of chemo-drug can protect normal tissues from toxicity and direct exposure.

In numerous pilot studies, microbubbles have been identified as a mechanism for encapsulating drugs, such as DOX and paclitaxel, for drug delivery [14-17]. The carried drug within bubbles could be triggered release upon US sonication, achieving local drug delivery while decreasing the drug exposure in blood circulation [18-22]. Additionally, bubbles exhibit a bi-functional characteristic that merges ultrasonic diagnosis with therapy. In this study, we aimed to build a DOX-loaded bubble (DOX-bubbles) system that provided: (1) a high drug (DOX) payload carrier with high ultrasonic sensitivity, (2) enhanced drug delivery triggered by US sonication, (3) ultrasonic imaging to assess tumor microperfusion information. This concurrent osteosarcoma theranostic strategy is shown in Figure 1. The fabrication and characteristics of DOX-bubbles, and their ability to improve cellular drug delivery with US is described herein. The feasibility of utilizing DOX-bubbles with contrast-enhanced ultrasound imaging (CEUS) for evaluating perfusion information of tumor was also assessed. Finally, the cancer treatment outcome with our proposed strategy was estimated using an animal OS tumor model, demonstrating the theranostic potential.

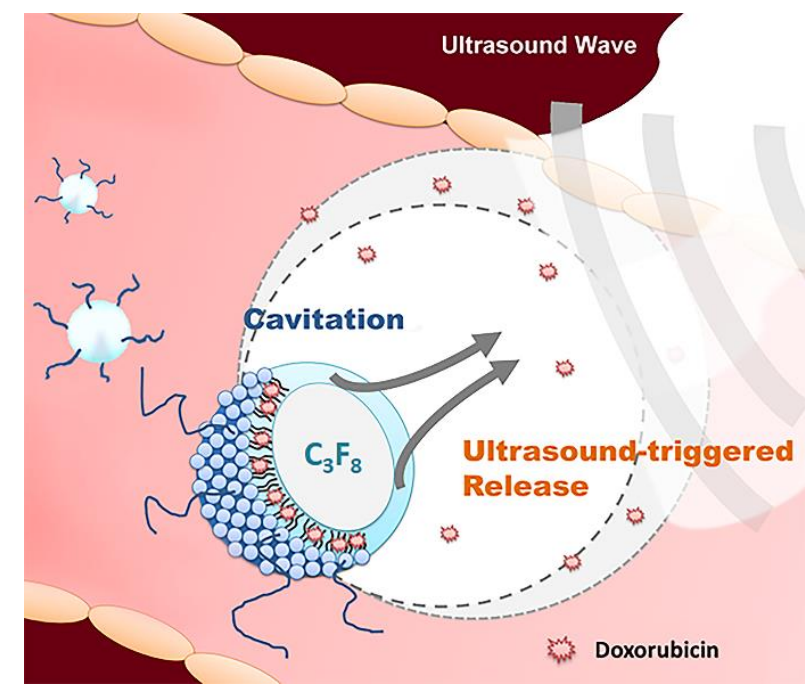

Figure 1. Diagram showing drug-loaded bubbles and ultrasound exposure to actively trigger drug release for osteosarcoma theranosis. 


\section{Material and Methods}

\subsection{Materials}

The pure lipid-based and perfluoropropane $\left(\mathrm{C}_{3} \mathrm{~F}_{8}\right)$-filled bubbles agent, was obtained in cooperation with Trust Bio-sonics, Inc. (Deliver, Hsinchu, Taiwan). The lipid shell consists primarily of 1,2-distearoyl-sn-glycero-3-phosphocholine (DSPC, Avanti Polar Lipids, Alabaster, AL, USA), 1,2-dioctadecanoyl-sn-glycero-3-phospho-(10-rac-glycerol) (DSPG, Avanti Polar Lipids, Alabaster, AL, USA), and 1,2-distearoyl-sn-glycero-3-phosphoethanolamine- $N$-[methoxy(poly(ethyleneglycol))-2000] (DSPE-PEG2000, Avanti Polar Lipids, Alabaster, AL, USA). The phosphate-buffered saline (PBS), glycerol, and doxorubicin (DOX) were purchased from Sigma-Aldrich (St. Louis, MO, USA).

\subsection{Preparation of Doxorubicin-Loaded Bubbles}

The molar ratio of DSPC, DSPG and DSPE-PEG2000 were 21:21:1. This information has been added in the revised manuscript (line). To load the bubbles with DOX molecules, a DOX aqua solution (with deionized water, in the concentration of $10 \mathrm{mg} / \mathrm{mL}$ ) was injected into pure bubbles without drug loading agent (Deliver) to mix with the formulated lipids [14,16]. The mixing ratio was based on the recommendation of $100 \mu \mathrm{L}$ DOX solution mixed with $400 \mu \mathrm{L}$ lipid solution. The final DOX concentration of the lipid solution was $2 \mathrm{mg} / \mathrm{mL}$. To further incorporate the DOX molecules into the lipid membranes, the mixed solution was placed in a $60{ }^{\circ} \mathrm{C}$ water bath for $30 \mathrm{~min}$. The mixed solution was then cooled to room temperature and agitated using an amalgamator (AM-1, MONITEX Industrial Co., New Taipei City, Taiwan) for $60 \mathrm{~s}$ to produce the DOX-loaded bubbles. The sample was centrifuged at $1000 \mathrm{~g}$ for $3 \mathrm{~min}$ to isolate DOX-bubbles from free drug molecules. After centrifugation, the solution layer was removed and then re-filled with fresh PBS to re-suspend the bubbles. The pure bubbles without drug loading and commercially available microbubbles (SonoVue ${ }^{\mathrm{TM}}$, Bracco Diagnostics Inc., Milan, Italy) were used for comparison.

\subsection{Characterization of DOX-Loaded Bubbles}

The size distribution and concentration of DOX-bubbles, pure bubbles, and SonoVue ${ }^{\mathrm{TM}}$ were confirmed by a Multisizer 3 device (Beckman Coulter, Brea, CA, USA) [23-25]. The morphology of DOX-bubbles was verified by a fluorescence microscope (IX-71, Olympus, Shinjuku-ku, Tokyo, Japan).

The signal-to-noise ratio (SNR) of DOX-bubbles was used to determine the in vitro acoustic stability acquired by an ultrasonic imaging system $(7.5 \mathrm{MHz}$, model $\mathrm{t} 3000$, Terason, Middlesex, MA, USA). The DOX-bubbles were first loaded into a channel within a $2 \%$ agarose phantom. The channel of the phantom was constructed by embedding a dialysis tube (BD Corp., Franklin Lakes, NJ, USA; diameter: $1 \mathrm{~mm}$ ) before phantom congealment. Once the agar gel had congealed, the tube was withdrawn, then leaving a wall-less tubular void. The images were then obtained periodically with a time interval of $10 \mathrm{~min}$ under $37^{\circ} \mathrm{C}$ for $1 \mathrm{~h}$. The SNR value was estimated from the contrast region within B-mode image.

When microbubble was destructed by US, it was referred to as inertial cavitation, and would emit acoustic broadband noise during microbubble collapse. Therefore, a 15-MHz US transducer (V303, Olympus, Waltham, NY, USA) was used to acquire the acoustic emission signals generated from microbubble under 1-MHz US exposure (model HS-3031, HES, Tainan, Taiwan; element size: 12 mm, peak-negative pressure: $0.1-1.0 \mathrm{MPa}$ ) for estimating the inertial cavitation threshold of microbubble. The focal zone of 1-MHz US was with a width of $9.7 \mathrm{~mm}$ and a length of $23 \mathrm{~mm}$. The acquired signals were then translated to frequency spectra via fast Fourier transform with MATLAB (The Mathworks, Natick, MA, USA) to verified the occurrence of broadband noise. The resonance frequency of DOX-bubbles was evaluated according the previous method with peak-negative pressure of 0.1 MPa [26]. The leakage of DOX from DOX-bubbles was assessed through evaluating the fluorescent intensity of the DOX-bubble suspension at each time point using a plate reader system (Safire, Tecan, AG, Switzerland) at $596 \mathrm{~nm}$. 
The drug retention with DOX-bubbles was estimated using the ratio of DOX leakage amount to the initial DOX loading amount.

The encapsulated DOX concentration of each DOX-bubble sample was calculated based on the fluorescence intensity of DOX. Before the measurement, the samples were sonicated to rupture the bubble structures and to avoid the densely encapsulated DOX molecules self-quenched in the lipid membranes.

The acoustic pressures used in this study were measured using a polyvinylidene difluoride type hydrophone (model HGL-0085, ONDA, Sunnyvale, CA, USA; calibration range: 1-40 MHz; spatial resolution: $85 \mu \mathrm{m}$ ) in an acrylic water tank that was filled with distilled and degassed water at $25^{\circ} \mathrm{C}$.

\subsection{In vitro Anti-Tumor Effect of DOX-Bubbles and Setting up the US Sonication System}

The MG-63 (CRL-1427) human osteosarcoma cell line was purchased from American Tissue Culture Collection (ATCC, Rockville, MD, USA) and cultured with Dulbecco's modified Eagles medium (HG-DMEM) and 10\% FBS. Prior to starting the experiment, $10^{4}$ of cells were seeded in a 96-well plastic plate (96-well Microtest ${ }^{\mathrm{TM}}$ Plate, BD Falcon ${ }^{\mathrm{TM}}$, Corning, NY, USA) and incubated with $5 \% \mathrm{CO}_{2}$ in $37^{\circ} \mathrm{C}$.

Normal saline, DOX, and DOX-bubbles were co-cultured with the cells for $20 \mathrm{~min}$ followed by 1-MHz US sonication (peak-negative pressure: $0.3 \mathrm{MPa}$, pulse repetition frequency: $1 \mathrm{~Hz}$, pulse length: $10 \mathrm{~ms}$, duty cycle: $1 \%$, time: $1 \mathrm{~min}$ ). So as to prevent the formation of standing wave, a plate made by ultrasound absorption material was placed on the bottom of cell plate during the treatment. After a 20-min incubation period, the cells were washed and refilled with fresh media. The experimental setup is illustrated in Figure 2A. Cytotoxicity was estimated using Alamar Blue (AbDSerotec, Oxford, UK). For determining the transmitted waveform and the acoustic pressure of this system, a 1-MHz transducer was calibrated using a hydrophone (model HGL-0085, ONDA, Sunnyvale, CA, USA) at $25^{\circ} \mathrm{C}$.

(A)

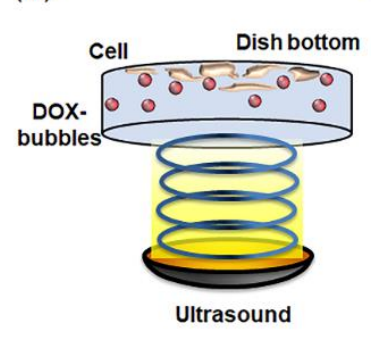

(B)

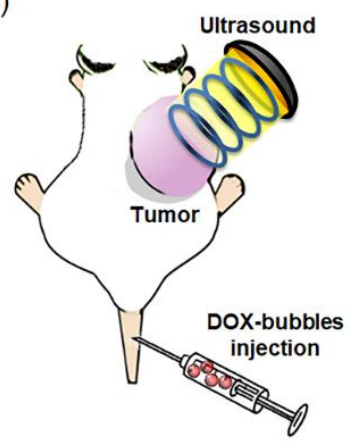

(C)

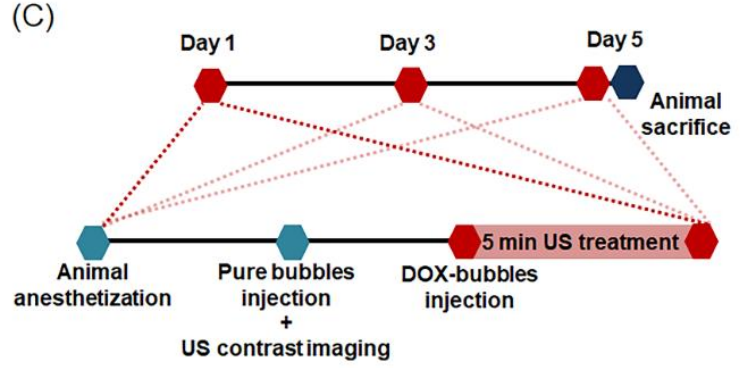

Figure 2. Experimental setup of (A) cell experiment and (B) animal tumor treatment. (C) Diagram of the osteosarcoma animal tumor treatment protocol. 


\subsection{In vivo Osteosarcoma Treatment}

\subsubsection{In-situ Osteosarcoma Tumor Model}

The immune-deficient NU-Foxn1nu mice used in our study protocols were purchased from the LASCO Laboratory (Taipei, Taiwan) and further cloned in specific pathogen-free condition at the Taipei Veterans General Hospital Animal Facility (Taipei, Taiwan) under the approved animal welfare and steps (IACUC 2012-188). Each mouse was IM injected with MG-63 cells through the right tibia from the knee joint for $1 \times 10^{7}$ cells/0.1 mL PBS at 8 weeks of age [27]. Before starting experiment, mice were anesthetized by IP injecting $50 \mu \mathrm{L}$ of Zoletil 50 (Virbac, Carros, France)/Rompun (Bayer HealthCare, Leverkusen, Germany) the mixed solution (50:50 vol\%).

\subsubsection{Ultrasound Imaging and Evaluation of Tumor Microcirculation}

To validate the capability of the DOX-bubbles in tumor microcirculation enhancement, a three-dimensional (3D) RSP6-16D transducer-equipped Voluson E8 ultrasound (GE healthcare, Ireland, UK) was used to obtain power Doppler volume histograms that were automatically calculated into indexes of vascularity (VI), flow (FI), and perfusion (PI). VI, represents the ratio of color-coded voxels to all voxels in the tumor. FI is the mean value of all color-coded voxels in the vessels of the volume analyzed. PI represents the mean value of voxels within the tumor region. The value of each color-coded voxel is expressed by the US instrument in arbitrary units (AU) on a scale of 0 to 100. These indexes were detected by DOX-bubbles and were further compared with values from pure bubbles and SonoVue ${ }^{\mathrm{TM}}$ to comprehend the effect of bubble size on microcirculation enhancement. During the comparisons, each animal received DOX-bubbles, pure bubbles, and SonoVue ${ }^{\mathrm{TM}}$ sequentially. The 3D Doppler scanning processes were performed at a mechanical index of 0.08 so as to avoid bubble destruction during the continuous scanning, and we also changed the injection order of these agents in each comparison to minimize the variance. The measurement was finished within $10 \mathrm{~min}$.

\subsubsection{Osteosarcoma Treatment Procedures}

Each treatment was initiated when the tumor volume reached $0.5 \mathrm{~cm}^{3}$. Three groups were evaluated including a control without treatment group ( $\mathrm{N}$-control, $\mathrm{N}=6$ ), DOX-bubble only group (DOX-bubbles only, $\mathrm{N}=6$ ), and a group that received DOX-bubbles with US insonation (DOX-bubbles $+\mathrm{US}, \mathrm{N}=6$ ). The distance between the transducer surface and tumor was set at $15 \mathrm{~mm}$. Before starting the treatment, each mouse underwent tumor microcirculation detection by pure bubbles $(30 \mu \mathrm{L})$ with CEUS. Thirty minutes later, the mice were treated using DOX-bubbles $(30 \mu \mathrm{L})$ with US. The time intervals between imaging and treatment allowed the bubbles to mostly clear from the circulation. The experimental setup and protocol are shown in Figure 2B,C. The tumor-bearing animal was placed prone, directly under a water cone with a $15 \times 15 \mathrm{~mm}^{2}$ window on the bottom, sealed with a polyurethane membrane to allow the entry of US treatment pulse. The tumor was tightly attached to the membrane window. A US coupling gel was applied between the tumor and the membrane to maximize the transmission of US between the transducer and the brain. The treatment repeated 3 times at day 1,3, and 5. The parameters of US treatment were set as previously described (Section 2.4). All insonations were set at $5 \mathrm{~min}$ and performed under the use of ultrasound gel to couple with the acoustic impedance.

\subsubsection{Histological Observation}

Histological observation was utilized to monitor the morphology of the tumor tissues from different groups. The tumor tissues were harvested after finishing the imaging evaluation on day 5 . Cryosection of the tissues was then performed at $20 \mu \mathrm{m}$ thickness, and sections were mounted on glass slides. After the preparation, the slides were stained with hematoxylin and eosin (H\&E stain, Sigma-Aldrich, St. Louis, MO, USA). 


\subsection{Statistics}

All results are represented as the mean \pm standard deviation. All statistical evaluations were carried out with unpaired two-tailed Student's t-tests. A $p$ value of less than 0.05 was referred to a significant difference.

\section{Results}

\subsection{Characteristics of DOX-bubbles}

The high co-localization of the bubbles, morphology and DOX fluorescence distribution suggests a successful combination of DOX and bubbles (Figure 3A). The mean size and concentration of the pure bubbles were $1.0 \pm 0.2 \mu \mathrm{m}$ and $33.2 \pm 1.4 \times 10^{9}$ bubbles $/ \mathrm{mL}$, respectively. The loading of DOX slightly enlarged the bubble size $(1.1 \pm 0.3 \mu \mathrm{m})$, and decreased the concentration $\left(18.7 \pm 5.9 \times 10^{9} \mathrm{MBs} / \mathrm{mL}\right)$ (Figure 3B). For comparison, the mean size and concentration of SonoVue were $5.6 \pm 0.9 \mu \mathrm{m}$ and $6.6 \pm 2.4 \times 10^{8}$ bubbles $/ \mathrm{mL}$, respectively. The DOX loading efficiency was $69.4 \pm 9.0 \%$, and the final loaded DOX was around $1.38 \mathrm{mg} / \mathrm{mL}$.

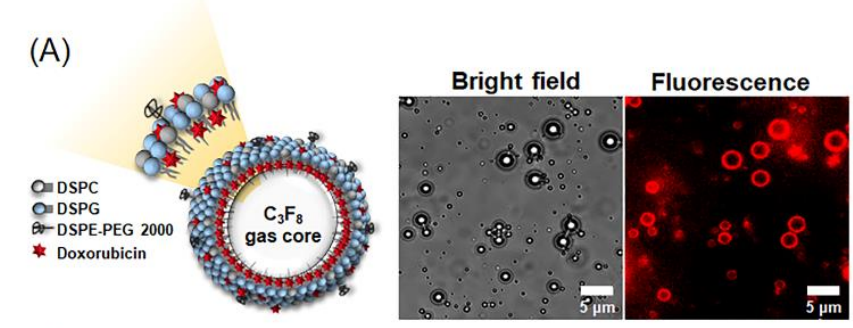

(B)

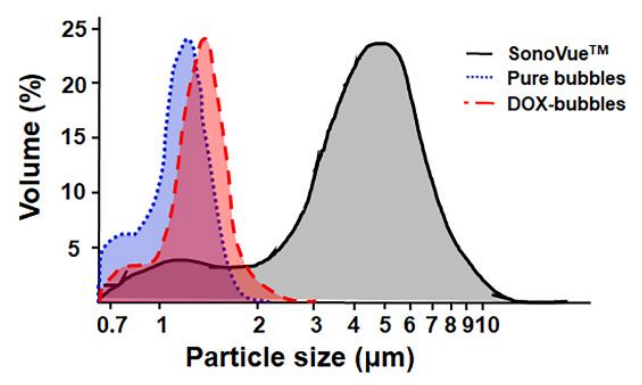

Figure 3. Size distribution and morphology of DOX (doxorubicin)-bubbles. (A) Left: the of DOX-bubbles; right: the bright field and fluorescent images of DOX-bubbles. Both of the images show the sphere shape of the DOX-bubbles, and the fluorescent image indicates the DOX molecules were incorporated in the lipid membranes. (B) Size distribution of pure bubbles, SonoVue $\mathrm{T}^{\mathrm{TM}}$, and DOX-bubbles.

The pure bubbles demonstrated stability over a $24 \mathrm{~h}$ period (size: from $1.0 \pm 0.1$ to $1.2 \pm 0.8 \mu \mathrm{m}$; concentration: from $(41.4 \pm 2.8) \times 10^{9}$ bubbles $/ \mathrm{mL}$ to $(42.9 \pm 1.3) \times 10^{9}$ bubbles $\left./ \mathrm{mL}\right)$ (Figure $\left.4 \mathrm{~A}\right)$. The size of DOX-bubbles showed stability at $4 \mathrm{~h}$ (from $1.1 \pm 0.1$ to $1.3 \pm 0.1 \mu \mathrm{m}$ ) that significantly increased at $24 \mathrm{~h}$ $(1.8 \pm 0.1 \mu \mathrm{m})$. The concentration of DOX-bubbles started decreasing at $30 \mathrm{~min}$ from $90 \%$ ((34.6 \pm 0.5$)$ $\times 10^{9}$ bubbles $\left./ \mathrm{mL}\right)$ to $20 \%\left((8.2 \pm 4.2) \times 10^{9}\right.$ bubbles $/ \mathrm{mL}$ after $24 \mathrm{~h}$ (Figure $\left.4 \mathrm{~B}\right)$. DOX leakage started from $10.4 \pm 9.8 \%$ at $2 \mathrm{~h}$ to $18.7 \pm 0.6 \%$ at $3 \mathrm{~h}$ and $98.2 \pm 12.5 \%$ after $24 \mathrm{~h}$ (Figure $4 \mathrm{C}$ ). Figure $4 \mathrm{D}$ shows the resonance frequency of DOX-bubbles was about $11-19 \mathrm{MHz}$. The acoustic stability of DOX-bubble remained relatively high until $20 \mathrm{~min}(0 \mathrm{~min}: 15.8 \pm 0.1 \mathrm{~dB} ; 30 \mathrm{~min}: 12.2 \pm 0.5 \mathrm{~dB} ; 1 \mathrm{~h}: 10.1 \mathrm{~dB} \pm 0.6 \mathrm{~dB}$ ). Since DOX delivery from DOX-bubbles needs the destruction of the DOX-bubbles by US, the US destruction threshold of DOX-bubbles was estimated. The inertial cavitation of DOX-bubbles was appeared when the acoustic pressure of US up to $0.3 \mathrm{MPa}$, indicating the onset of DOX-bubble collapse (Figure 4F). There were no differences in the destruction threshold between DOX-bubbles and pure 
bubbles. Therefore, we used 0.3 MPa of US sonication for the following experiments. These data also concluded that the properties of the bubbles were not affected by the encapsulation of DOX.

(A)

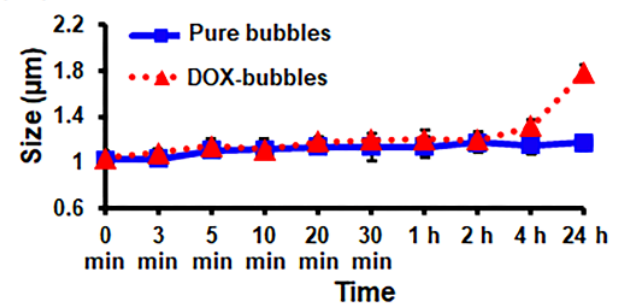

(B)

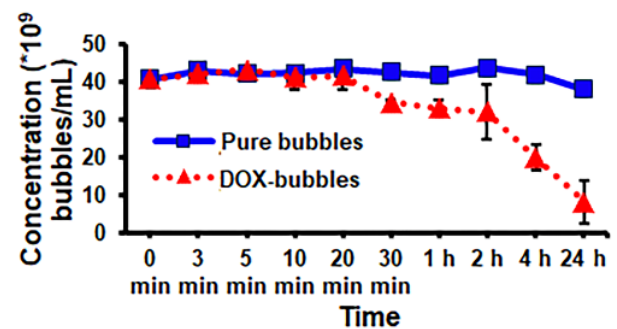

(C)

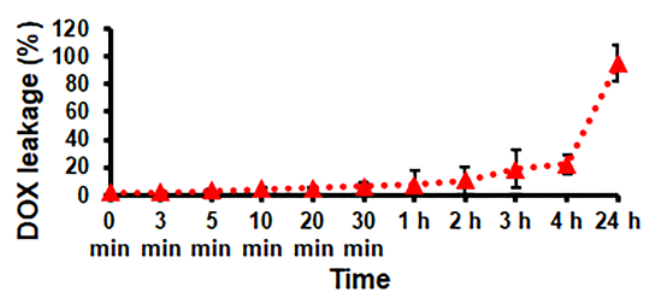

(D)

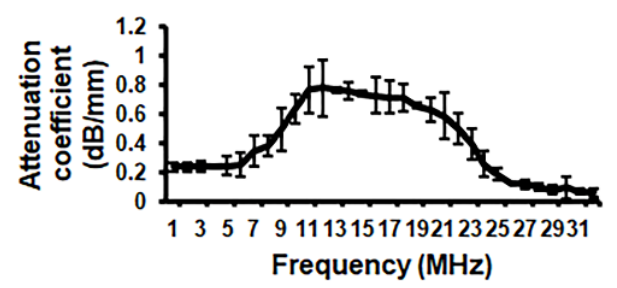

(E)

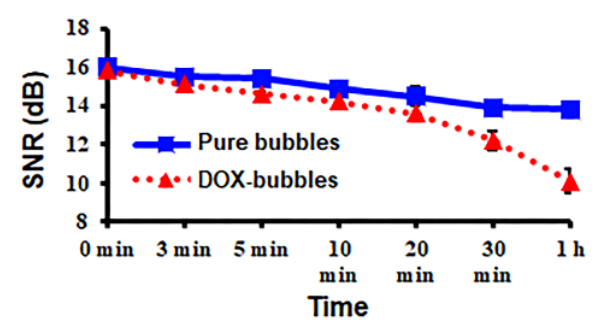

( $F)$

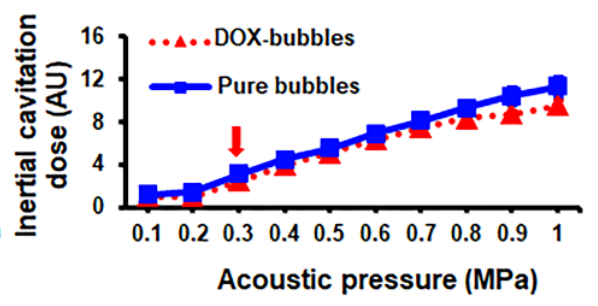

Figure 4. Properties of DOX-bubbles and pure bubbles. Size distribution (A) and concentration (B) of the two bubbles measured via Coulter counter at $37^{\circ} \mathrm{C}$ at different time point. (C) Leakage of DOX from DOX-bubbles at $37^{\circ} \mathrm{C}$ at different time points. (D) Attenuation measurements representing the resonance of DOX-bubbles with respect to the frequency of ultrasonic exposure. (E) In vitro acoustic stability of DOX-bubbles and pure bubbles. (F) The acoustic destruction threshold of DOX-bubbles and pure bubbles.

\subsection{Controlled DOX Intracellular Delivery by DOX-Bubbles with US}

Next, the controlled drug release capability of DOX-bubbles upon US exposure was investigated in MG-63 cells. The fluorescent images confirmed the intracellular deposition of DOX in DOX-bubbles + US group, indicating that the encapsulated DOX could be triggered delivery into cells in conjunction with US exposure (Figure 5A). Cell viability was unaffected when US was applied alone. Administration with DOX alone caused a lower cell viability $(52.5 \pm 7.9 \%)$. DOX-bubble incubation alone produced a minor decrease in cell viability $(85.5 \% \pm 4.4 \%)$, likely because of the natural drug leakage from DOX-bubbles. The cytotoxicity of DOX could also be restricted by the protection of bubbles. Nevertheless, the combination between DOX-bubbles and US sonication also could provide anti-tumor ability $(33.7 \pm 9.9 \%)$ (Figure $5 \mathrm{~B}$ ), indicated that the DOX released from DOX-bubbles still had cytotoxicity. These data suggest that the DOX embedded in DOX-bubbles could be triggered for release by US so as to kill off the tumor cells. 


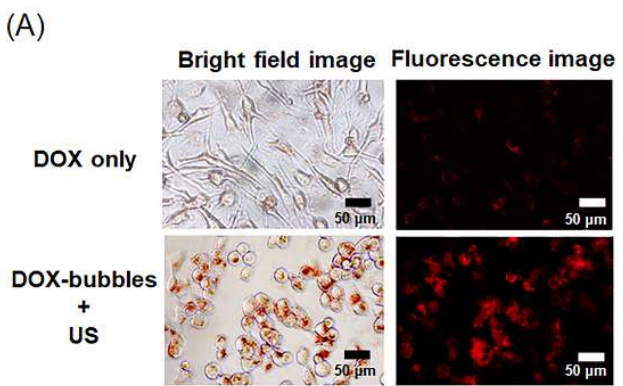

(B)

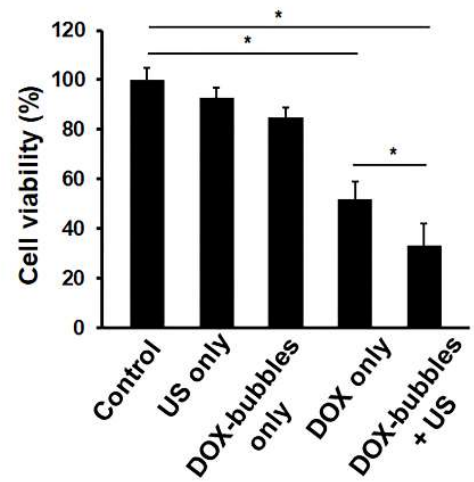

Figure 5. In vitro antitumor effect of DOX-bubbles and US (ultrasound) sonication. (A) Bright-field and fluorescent images of MG-63 cells treated with DOX, and DOX-bubbles + US. (B) Cell viability after treatment. Single asterisk, $p<0.05$.

\subsection{In vivo Monitoring of Tumor Microcirculation Perfusion and Volume by DOX-Bubbles with CEUS}

Next, we evaluated the tumor microcirculation enhancement of DOX-bubbles with CEUS. The SonoVue ${ }^{\mathrm{TM}}$ was used as the current standard for comprehending the effect of bubble size on microcirculation detection. Note that the concentrations of these bubbles were different (pure-bubbles: $33.2 \pm 1.4 \times 10^{9}$ bubbles $/ \mathrm{mL}$, DOX-bubbles: $18.7 \pm 5.9 \times 10^{9}$ bubbles $/ \mathrm{mL}$, SonoVue: $6.6 \pm 2.4 \times 10^{8}$ bubbles/mL), so we injected these bubbles with different volumes to ensure that the number of bubbles in each group was the same. The final injected volume of pure-bubbles, DOX-bubbles and SonoVue ${ }^{\mathrm{TM}}$ were $20 \mu \mathrm{L}, 35 \mu \mathrm{L}$ and, $100 \mu \mathrm{L}$, respectively. Figure $6 \mathrm{~A}$ shows the contrast signal intensity increment after administrating DOX-bubbles, and the same imaging technique could be further combined with 3D scanning to illustrate the volume information. These results suggest that compared with the SonoVue ${ }^{\mathrm{TM}}$, both pure bubbles and DOX-bubbles showed a better microcirculation enhancement. The VI, FI, and PI of DOX-bubbles were $70.4 \pm 2.8 \%, 67.1 \pm 6.1 \mathrm{AU}$, and $13.1 \pm 2.9 \mathrm{AU}$, respectively (Figure 6B). The VI, FI, and PI of pure bubbles were $75.9 \pm 8.8 \%, 76.9 \pm 10.5 \mathrm{AU}$, and $14.4 \pm 3.3 \mathrm{AU}$, respectively. For the current standard, SonoVue ${ }^{\mathrm{TM}}$, the VI, FI, and PI were $39.8 \pm 3.8 \%, 45.2 \pm 3.3 \mathrm{AU}$, and $4.9 \pm 1.5 \mathrm{AU}$, respectively. All the differences between these three indexes between DOX-bubbles and SonoVue ${ }^{\mathrm{TM}}$ were statistically significant. There were no differences in these three indexes between DOX-bubbles and pure bubbles. This result indicates that the DOX-bubbles could be a reliable contrast media for tumor microcirculation enhancement and volume detection since it provided the missing information when a typical larger sized agent is used. Further, it might have the functionality to identify a small change in tumor vascularity before and after chemotherapies or the purposed concurrent OS theranostic strategy in this study. 


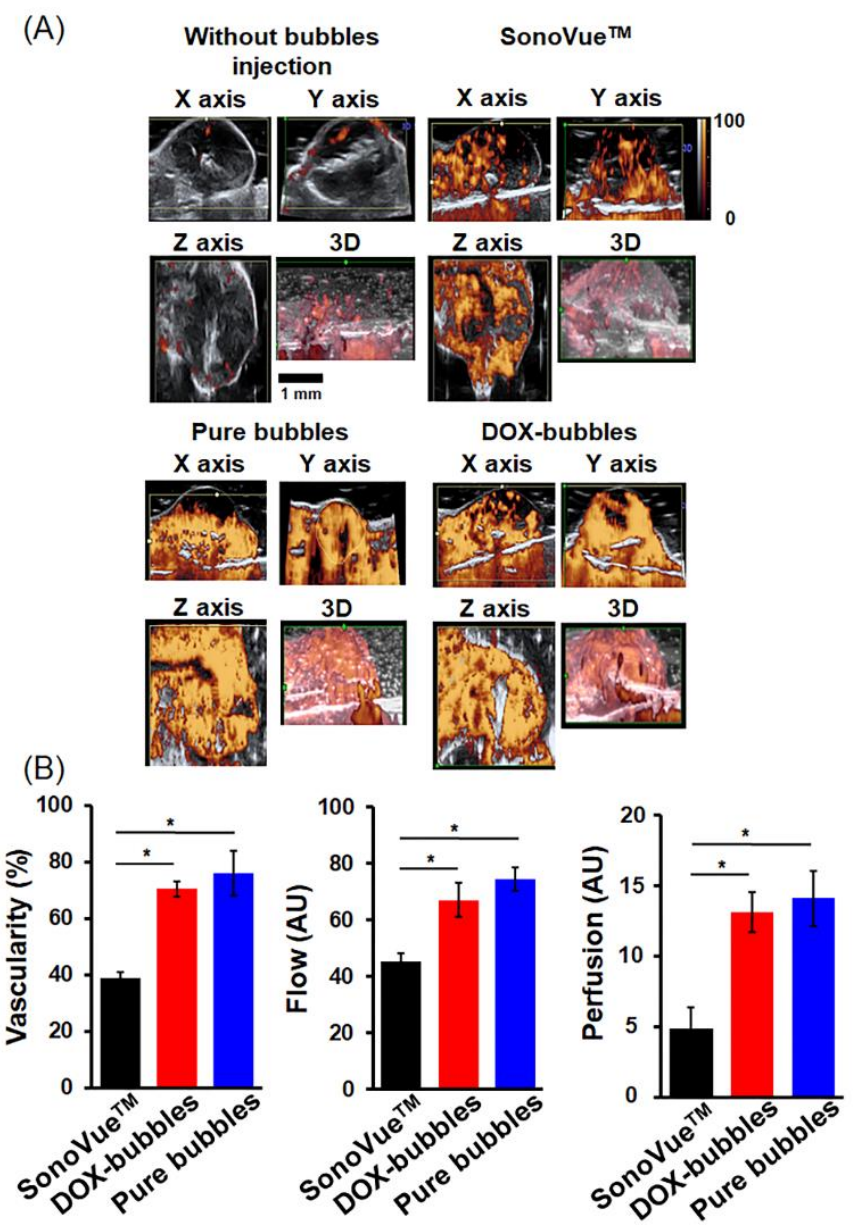

Figure 6. (A) The Doppler ultrasound images of osteosarcoma tumor model. Three groups were involved, without bubbles injection, SonoVue ${ }^{\mathrm{TM}}$, pure bubbles, and DOX-bubbles. Note that the scale bar was for all the sub-figures. (B) Left: vascularity index (VI), denotes the ratio of color-coded voxels to all voxels of the tumor region; middle: flow index (FI), denotes the mean value of all color-coded voxels in the vessels of the volume analyzed; right: perfusion index (PI), denotes the mean value of voxels within the tumor region. These results suggested that compared with the SonoVue ${ }^{\mathrm{TM}}$, DOX-bubbles showed better microcirculation enhancement. Single asterisk, $p<0.05$.

\subsection{In vivo OS Treatment Outcome and Concurrent Theranostic Strategy with DOX-Bubbles}

The therapeutic efficacy results are shown in Figure 7A. The normalized therapeutic efficacy is defined as the mean volume change between the experimental group and negative control. Only the group of DOX-bubbles + US could provide a significant suppression of tumor growth (day 1: $0.4 \pm 0.1 \mathrm{~cm}^{3}$; day 3: $1.3 \pm 0.7 \mathrm{~cm}^{3}$; day $\left.5: 1.8 \pm 0.9 \mathrm{~cm}^{3}\right)$, and the normalized therapeutic efficacy was increased 3.7-fold compared with the group without treatment (N-control group, day 1: $0.5 \pm 0.3 \mathrm{~cm}^{3}$, day 3: $3.9 \pm 0.9 \mathrm{~cm}^{3}$, day 3: $8.5 \pm 2.2 \mathrm{~cm}^{3}$ vs. DOX-bubbles only group, day 1: $0.4 \pm 0.1 \mathrm{~cm}^{3}$, day 3: $2.6 \pm 0.9 \mathrm{~cm}^{3}$, day 5: $6.5 \pm 2.4 \mathrm{~cm}^{3}$ ). We also compared the overall perfusions of all groups between day 1 and day 5 to investigate the feasibility of using CEUS to evaluate the therapeutic outcome. Figure 7B demonstrates that the treatment of DOX-bubbles + US resulted in a significant decline of blood flow in the tumor area, suggesting the occurrence of DOX-induced necrosis. We also noticed an interesting trend, that only the perfusion of N-control group increased within 5 days from $10.1 \pm 3.8$ AU to $15.2 \pm 4.9 \mathrm{AU}$. The overall perfusions of both groups that received DOX-bubbles were reduced from $12.1 \pm 2.1$ to $9.6 \pm 3.4 \mathrm{AU}$ for the group of DOX-bubble only group, and $11.3 \pm 3.2$ to $4.3 \pm 1.9$ $\mathrm{AU}$ for the group of DOX-bubbles + US. The macroscopic and histological observations shown in Figure $7 \mathrm{C}$ also agreed with the imaging data. These outcomes provide evidence that the synergistic 
effect of DOX-bubbles with US could contribute to suppression of tumor growth and acceleration of tumor necrosis. Furthermore, the therapeutic outcome could be monitored by pure bubbles with CEUS, suggesting the theranostic application of our proposed DOX-bubbles.
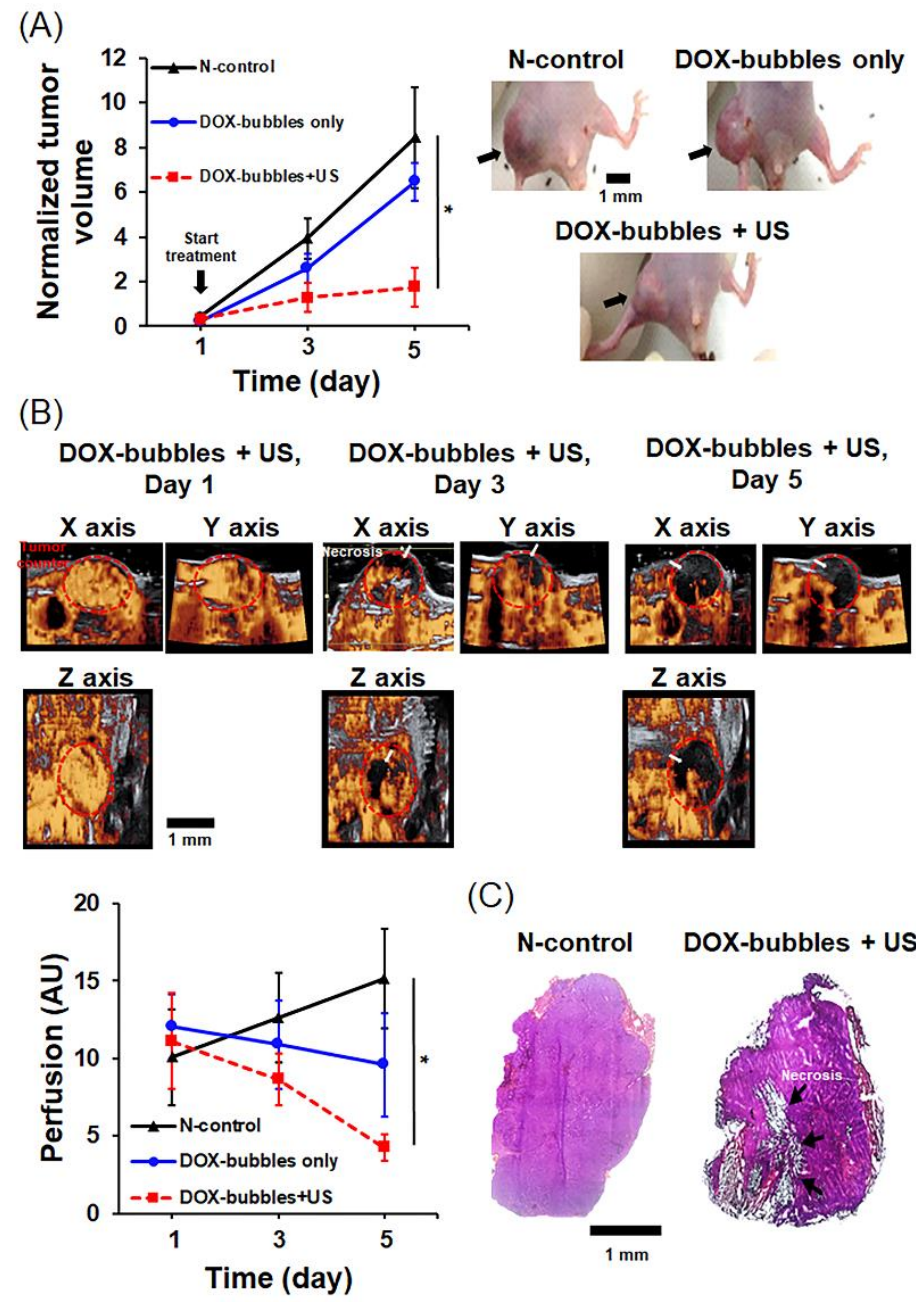

(C)
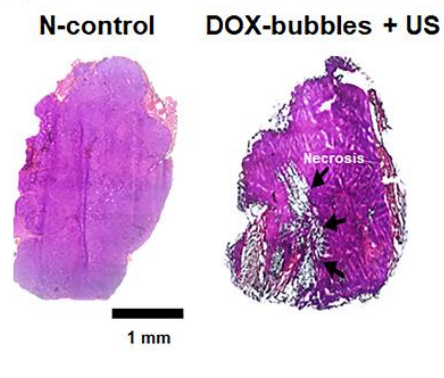

Figure 7. (A) Left: the therapeutic efficacy on the suppression of tumor volume; right: The macroscopic observation of the osteosarcoma tumor models of three groups. (B) The contrast-enhanced ultrasound imaging (CEUS) images (upper) and the tumor perfusion (bottom) before and after treatments with pure bubbles injection (the largest tumor section obtained from each scanning). White arrow: necrotic regions. Red dotted region: tumor contour. (C) Histological observation of the tumor after treatment. Black arrows: necrotic regions. Single asterisk, $p<0.05$.

\section{Discussion}

\subsection{Significance}

We showed the efficacious use of DOX-loaded bubbles for transporting DOX in combination with US sonication as a theranostic approach for OS treatment. The fabricated DOX-bubbles showed better tumor microcirculation enhancement than commercial bubble SonoVue ${ }^{\mathrm{TM}}$ with CEUS imaging. Additionally, the tumor growth was suppressed by treatment with DOX-bubbles and US in only five days because of the acceleration of the DOX entering to tumor cells and leading necrosis process. This study provides a new non-invasive approach for delivering chemotherapeutic drug in OS treatment. 


\subsection{Preparation of DOX-Bubbles}

The chemotherapeutic drug (DOX) loading onto the lipid shells of bubbles relies on electrostatic and hydrophobic interactions. The red fluorescence of DOX-bubbles revealed successful complexation of the cationic microbubbles with DOX (Figure 3). Our data conclude that the stability and acoustic property of bubbles were not affected by the encapsulation of DOX. In addition, the cytotoxicity of DOX could be reduced after the encapsulation into bubbles, regardless of US irradiation. On the other hand, the size of bubbles did not obviously change before and after DOX loading (pure bubbles: $1.0 \pm 0.2 \mu \mathrm{m}$ vs. DOX-bubbles: $1.1 \pm 0.3 \mu \mathrm{m}$ ). It was reported that the microbubbles might be aggregated forming large clusters or coalesce into larger bubbles during long-pulsed US stimulation [28-30]. These effects might induce gas embolism within circulation, blocking local blood flow and decreasing drug delivery efficiency. Future works should verify the occurrence of these effects during treatment and adjust the US treatment parameters to control the clustering process. The small size of the DOX-bubbles produced better tumor microcirculation enhancement as well as potentially increased the safety during treatment. Previous reports have indicated that the bio-effects from bubbles could be reduced by decreasing the size of the bubbles because larger bubbles can easily expand and fragment while in contact with the endothelial cells [31,32].

\subsection{US Treatment}

Previous studies have demonstrated that ultrasound exposure can trigger drug release from drug-loaded bubbles and also enhance drug permeability of tumor vessels through the cavitation effect [33,34]. This effect might be the most important difference between free drugs and the bubble-based drug delivery systems. The disruption of bubbles caused both inertial and stable cavitation to induce micro-streaming and liquid jets. These mechanical forces stimulate shrinkage of vessels and increased drug permeability [35-37]. To avoid the heating effect from high-intensity and continuous insonation, we used low-intensity $(\mathrm{MI}=0.3)$ and pulsed (pulse repetition frequency $=1 \mathrm{~Hz}$ ) US insonation parameters to perform the treatment. Thus, we believe that the therapeutic outcome in the experimental group of DOX-bubbles + US was driven by chemotherapeutics and stimulation. Note that pulsed ultrasound is used to avoid the heating effect of ultrasound and provide a sufficient time interval for the reperfusion of DOX-bubbles $[38,39]$. Through a by-side ultrasound imaging system, we monitored the reperfusion of the DOX-bubbles for estimating the treatment outcome. However, the results also indicated that ultrasound insonations are needed since only the group of DOX-bubbles + US significantly suppressed tumor growth. Therefore, physicians can probably use the therapeutic DOX-bubbles to simultaneously perform both perfusion evaluation and treatment in a real clinical setting. Thus, DOX-bubbles can be used as a theranostic agent for concurrent osteosarcoma theranosis. Conversely, previous studies had reported the lifetime of microbubbles and the spatiotemporal uniformity of cavitation activity by short-pulse ultrasound $[40,41]$, suggesting that the distribution of cavitation perhaps could be adjusted by the waveform of US. Therefore, the future work includes resulting in efficient therapies by spreading cavitation throughout the treatment area with code-excitation US pulse.

\subsection{Limitations}

The limitations of the purposed strategy might lie on the penetration depth of ultrasound. To demonstrate the attenuation of 1-MHz ultrasound, we measured the attenuated acoustic intensity during penetration of a 5-mm-thick pork humerus, and the result showed that the acoustic pressure would be reduced to $0.1 \mathrm{MPa}$ (refers to $7.13 \mathrm{~dB}$, data not shown). In contrast to $1-\mathrm{MHz}$ ultrasound, we also tested a 3-MHz transducer using the same setups, and the acoustic pressure was dramatically reduced from 1.1 $\mathrm{MPa}$ to $0.1 \mathrm{MPa}$ (refers to $20.83 \mathrm{~dB}$, data not shown). The inertial cavitation threshold of bubbles is typically around 0.1 to $0.2 \mathrm{MPa}$ when using low frequency ultrasound (less than $3 \mathrm{MHz}$ ). This comparison reveals that lower frequencies (lower than $1 \mathrm{MHz}$ ) or higher intensity (higher than $1 \mathrm{MPa}$ ) might be more useful for osteosarcoma treatments. The new technology, high-intensity focused 
ultrasound (HIFU), which is now currently used to ablate the bone tumors [42-44], has become more popular in recent years. The local acoustic pressure of HIFU could be heightened to more than $10 \mathrm{MPa}$ for direct penetration into tumor tissues. Thus, the combination of DOX-bubbles and HIFU might be another way to perform concurrent osteosarcoma theranosis. However, planar ultrasound is probably more suitable for regional treatments than for larger insonation areas. As a result, a planar ultrasound device that can transmit high-intensity ultrasound is critical for launching a concurrent osteosarcoma theranostic strategy in a clinical setting. Another limitation of the current study was the absence of flow in the in vitro experiments. The blood flow would affect the microbubble dynamics and the pressure thresholds required to achieve intracellular delivery [45]. Besides, the in vitro experiments were conducted by monolayer cell structure, making it was hard to refer to tumor conditions. Future works include designing an in vitro flow system or tumor-chip device for observing the potential mechanisms of US + drug-loaded bubbles regulated drug delivery under flow condition.

\section{Conclusions}

In this study, we established an approach using drug-loaded bubbles for concurrent osteosarcoma theranosis. The combination of low-intensity $(0.3 \mathrm{MPa})$ US and drug-loaded bubbles provides an efficient controlled release strategy for providing 3.7-fold suppression of tumor growth compared with the group without US exposure $\left(1.8 \pm 0.9 \mathrm{~cm}^{3}\right.$ vs. $\left.8.5 \pm 2.2 \mathrm{~cm}^{3}\right)$. Using CEUS technology, we also found that the proposed drug-loaded bubbles showed 1.8-fold of vascularity $(70.4 \pm 2.8 \% \mathrm{vs}$. $39.8 \pm 3.8 \%), 1.7$-fold of flow $(67.1 \pm 6.1 \mathrm{AU}$ vs. $45.2 \pm 3.3 \mathrm{AU})$, and 2.7 -fold of perfusion $(13.1 \pm 2.9 \mathrm{AU}$ vs. $4.9 \pm 1.5 \mathrm{AU}$ ) higher than commercialized microbubbles in tumor microcirculation detection. This study provided a novel theranostic strategy for US regulated tumor drug delivery into clinical use.

Author Contributions: T.-T.K., C.-H.F., and C.-K.Y. contributed for the manuscript preparation. T.-T.K. and C.-K.Y. designed the experiment. T.-T.K. summarized and analyzed data. C.-H.W. performed the animal study. J.-Y.W. and H.-J.C. provided the experimental equipment.

Funding: This research was funded by Ministry of Science and Technology, Taiwan, grants number 107-2221-E-007-002, 107-2627-M-007-005, and by National Tsing Hua University (Hsinchu, Taiwan), grants number 107Q2717E1, and by Taipei Veterans General Hospital (Taipei, Taiwan), grant number VGH-103-C-121.

Conflicts of Interest: The authors declare no conflicts of interest.

\section{References}

1. Sluga, M.; Windhager, R.; Pfeiffer, M.; Ofner, P.; Lang, S.; Dominkus, M.; Nehrer, S.; Zoubek, A.; Kotz, R. Osteosarcoma and Ewing's sarcoma-The most frequent malignant bone tumors in children-therapy and outcome. Z. Orthop. Ihre Grenzgeb. 2002, 140, 652-655. [CrossRef] [PubMed]

2. He, A.; Yang, X.; Huang, Y.; Feng, T.; Wang, Y.; Sun, Y.; Shen, Z.; Yao, Y. CD133(+) CD44(+) Cells Mediate in the Lung Metastasis of Osteosarcoma. J. Cell Biochem. 2015, 116, 1719-1729. [CrossRef] [PubMed]

3. Torre, L.A.; Bray, F.; Siegel, R.L.; Ferlay, J.; Lortet-Tieulent, J.; Jemal, A.N.; aishadham, D.; Jemal, A. Cancer statistics, 2013. CA Cancer J. Clin. 2013, 65, 87-108. [CrossRef] [PubMed]

4. Li, S.; Sun, W.; Wang, H.; Zuo, D.; Hua, Y.; Cai, Z. Research progress on the multidrug resistance mechanisms of osteosarcoma chemotherapy and reversal. Tumour Biol. 2015, 36, 1329-1338. [CrossRef] [PubMed]

5. Schwartz, C.L.; Gorlick, R.; Teot, L.; Krailo, M.; Chen, Z.; Goorin, A.; Grier, H.E.; Bernstein, M.L.; Meyers, P. Multiple drug resistance in osteogenic sarcoma: INT0133 from the Children's Oncology Group. J. Clin. Oncol. 2007, 25, 2057-2062. [CrossRef] [PubMed]

6. Thorn, C.F.; Oshiro, C.; Marsh, S.; Hernandez-Boussard, T.; McLeod, H.; Klein, T.E.; Altman, R.B. Doxorubicin pathways: Pharmacodynamics and adverse effects. Pharmacogenet. Genom. 2011, 21, 440-446. [CrossRef] [PubMed]

7. Chou, A.J.; Gorlick, R. Chemotherapy resistance in osteosarcoma: Current challenges and future directions. Expert Rev. Anticancer Ther. 2006, 6, 1075-1085. [CrossRef]

8. Wang, L.; Wang, W.; Rui, Z.; Zhou, D. The effective combination therapy against human osteosarcoma: Doxorubicin plus curcumin co-encapsulated lipid-coated polymeric nanoparticulate drug delivery system. Drug Deliv. 2016, 23, 3200-3208. [CrossRef] [PubMed] 
9. Hynynen, K.; McDannold, N.; Vykhodtseva, N.; Jolesz, F.A. Noninvasive MR imaging-guided focal opening of the blood-brain barrier in rabbits. Radiology 2001, 220, 640-646. [CrossRef]

10. Dimcevski, G.; Kotopoulis, S.; Bjanes, T.; Hoem, D.; Schjott, J.; Gjertsen, B.T.; Biermann, M.; Molven, A.; Sorbye, H.; McCormack, E.; et al. A human clinical trial using ultrasound and microbubbles to enhance gemcitabine treatment of inoperable pancreatic cancer. J. Control. Release 2016, 243, 172-181. [CrossRef]

11. Shohet, R.V.; Chen, S.; Zhou, Y.T.; Wang, Z.; Meidell, R.S.; Unger, R.H.; Grayburn, P.A. Echocardiographic destruction of albumin microbubbles directs gene delivery to the myocardium. Circulation 2000, 101, 2554-2556. [CrossRef]

12. Leong-Poi, H.; Kuliszewski, M.A.; Lekas, M.; Sibbald, M.; Teichert-Kuliszewska, K.; Klibanov, A.L.; Stewart, D.J.; Lindner, J.R. Therapeutic arteriogenesis by ultrasound-mediated VEGF165 plasmid gene delivery to chronically ischemic skeletal muscle. Circ. Res. 2007, 101, 295-303. [CrossRef] [PubMed]

13. Carson, A.R.; McTiernan, C.F.; Lavery, L.; Grata, M.; Leng, X.; Wang, J.; Chen, X.; Villanueva, F.S. Ultrasound-targeted microbubble destruction to deliver siRNA cancer therapy. Cancer Res. 2012, 72, 6191-6199. [CrossRef] [PubMed]

14. Fan, C.H.; Ting, C.Y.; Lin, H.J.; Wang, C.H.; Liu, H.L.; Yen, T.C.; Yeh, C.K. SPIO-conjugated, doxorubicin-loaded microbubbles for concurrent MRI and focused-ultrasound enhanced brain-tumor drug delivery. Biomaterials 2013, 34, 3706-3715. [CrossRef]

15. Ueno, Y.; Sonoda, S.; Suzuki, R.; Yokouchi, M.; Kawasoe, Y.; Tachibana, K.; Maruyama, K.; Sakamoto, T.; Komiya, S. Combination of ultrasound and bubble liposome enhance the effect of doxorubicin and inhibit murine osteosarcoma growth. Cancer Biol. Ther. 2011, 12, 270-277. [CrossRef] [PubMed]

16. Tinkov, S.; Winter, G.; Coester, C.; Bekeredjian, R. New doxorubicin-loaded phospholipid microbubbles for targeted tumor therapy: Part I-Formulation development and in-vitro characterization. J. Control. Release 2010, 143, 143-150. [CrossRef]

17. Yan, F.; Li, L.; Deng, Z.; Jin, Q.; Chen, J.; Yang, W.; Yeh, C.K.; Wu, J.; Shandas, R.; Liu, X.; et al. Paclitaxel-liposome-microbubble complexes as ultrasound-triggered therapeutic drug delivery carriers. J. Control. Release 2013, 166, 246-255. [CrossRef]

18. Ting, C.Y.; Fan, C.H.; Liu, H.L.; Huang, C.Y.; Hsieh, H.Y.; Yen, T.C.; Wei, K.C.; Yeh, C.K. Concurrent blood-brain barrier opening and local drug delivery using drug-carrying microbubbles and focused ultrasound for brain glioma treatment. Biomaterials 2012, 33, 704-712. [CrossRef]

19. Nesbitt, H.; Sheng, Y.; Kamila, S.; Logan, K.; Thomas, K.; Callan, B.; Taylor, M.A.; Love, M.; O’Rourke, D.; Kelly, P.; et al. Gemcitabine loaded microbubbles for targeted chemo-sonodynamic therapy of pancreatic cancer. J. Control. Release 2018, 279, 8-16. [CrossRef]

20. Logan, K.; Foglietta, F.; Nesbitt, H.; Sheng, Y.; McKaig, T.; Kamila, S.; Gao, J.; Nomikou, N.; Callan, B.; McHale, A.P.; et al. Targeted Chemo-Sonodynamic Therapy Treatment of Breast Tumours Using Ultrasound Responsive Microbubbles Loaded with Paclitaxel, Doxorubicin and Rose Bengal. Eur. J. Pharm. Biopharm. 2019, 139, 224-231. [CrossRef]

21. Sirsi, S.R.; Borden, M.A. State-of-the-art materials for ultrasound-triggered drug delivery. Adv. Drug Deliv. Rev. 2014, 72, 3-14. [CrossRef]

22. Dewitte, H.; Vanderperren, K.; Haers, H.; Stock, E.; Duchateau, L.; Hesta, M.; Saunders, J.H.; De Smedt, S.C.; Lentacker, I. Theranostic mRNA-loaded microbubbles in the lymphatics of dogs: Implications for drug delivery. Theranostics 2015, 5, 97-109. [CrossRef] [PubMed]

23. Wang, C.H.; Yeh, C.K. Controlling the size distribution of lipid-coated bubbles via fluidity regulation. Ultrasound Med. Biol. 2013, 39, 882-892. [CrossRef] [PubMed]

24. Feshitan, J.A.; Chen, C.C.; Kwan, J.J.; Borden, M.A. Microbubble size isolation by differential centrifugation. J. Colloid Interface Sci. 2009, 329, 316-324. [CrossRef]

25. Zhao, S.; Borden, M.; Bloch, S.H.; Kruse, D.; Ferrara, K.W.; Dayton, P.A. Radiation-force assisted targeting facilitates ultrasonic molecular imaging. Mol. Imaging 2004, 3, 135-148. [CrossRef]

26. Sun, Y.; Kruse, D.E.; Dayton, P.A.; Ferrara, K.W. High-frequency dynamics of ultrasound contrast agents. IEEE Trans. Ultrason. Ferroelectr. Freq. Control 2005, 52, 1981-1991. [PubMed]

27. Wang, J.Y.; Wu, P.K.; Chen, P.C.; Yen, C.C.; Hung, G.Y.; Chen, C.F.; Hung, S.C.; Tsai, S.F.; Liu, C.L.; Chen, T.H.; et al. Manipulation therapy prior to diagnosis induced primary osteosarcoma metastasis-from clinical to basic research. PLoS ONE 2014, 9, e96571. [CrossRef]

28. Lazarus, C.; Pouliopoulos, A.N.; Tinguely, M.; Garbin, V.; Choi, J.J. Clustering dynamics of microbubbles exposed to low-pressure 1-MHz ultrasound. J. Acoust. Soc. Am. 2017, 142, 3135-3146. [CrossRef] 
29. Postema, M.; van Wamel, A.; Lancée, C.T.; de Jong, N. Ultrasound-induced encapsulated microbubble phenomena. Ultrasound Med. Biol. 2004, 30, 827-840. [CrossRef]

30. Fan, Z.; Chen, D.; Deng, C.X. Characterization of the dynamic activities of a population of microbubbles driven by pulsed ultrasound exposure in sonoporation. Ultrasound Med. Biol. 2014, 40, 1260-1272. [CrossRef] [PubMed]

31. Choi, J.J.; Feshitan, J.A.; Baseri, B.; Wang, S.; Tung, Y.S.; Borden, M.A.; Konofagou, E.E. Microbubble-size dependence of focused ultrasound-induced blood-brain barrier opening in mice in vivo. IEEE Trans. Biomed. Eng. 2010, 57, 145-154. [CrossRef] [PubMed]

32. Samiotaki, G.; Vlachos, F.; Tung, Y.S.; Konofagou, E.E. A quantitative pressure and microbubble-size dependence study of focused ultrasound-induced blood-brain barrier opening reversibility in vivo using MRI. Magn. Reson. Med. 2012, 67, 769-777. [CrossRef]

33. Vlachos, F.; Tung, Y.S.; Konofagou, E. Permeability dependence study of the focused ultrasound-induced blood-brain barrier opening at distinct pressures and microbubble diameters using DCE-MRI. Magn. Reson. Med. 2011, 66, 821-830. [CrossRef] [PubMed]

34. Sheikov, N.; McDannold, N.; Sharma, S.; Hynynen, K. Effect of focused ultrasound applied with an ultrasound contrast agent on the tight junctional integrity of the brain microvascular endothelium. Ultrasound Med. Biol. 2008, 34, 1093-1104. [CrossRef]

35. Raymond, S.B.; Skoch, J.; Hynynen, K.; Bacskai, B.J. Multiphoton imaging of ultrasound/Optison mediated cerebrovascular effects in vivo. J. Cereb. Blood Flow Metab. 2007, 27, 393-403. [CrossRef]

36. Cho, E.E.; Drazic, J.; Ganguly, M.; Stefanovic, B.; Hynynen, K. Two-photon fluorescence microscopy study of cerebrovascular dynamics in ultrasound-induced blood-brain barrier opening. J. Cereb. Blood Flow Metab. 2011, 31, 1852-1862. [CrossRef]

37. Sheikov, N.; McDannold, N.; Vykhodtseva, N.; Jolesz, F.; Hynynen, K. Cellular mechanisms of the blood-brain barrier opening induced by ultrasound in presence of microbubbles. Ultrasound Med. Biol. 2004, 30, 979-989. [CrossRef] [PubMed]

38. Fan, C.H.; Lin, W.H.; Ting, C.Y.; Chai, W.Y.; Yen, T.C.; Liu, H.L.; Yeh, C.K. Contrast-enhanced ultrasound imaging for the detection of focused ultrasound-induced blood-brain barrier opening. Theranostics 2014, 4, 1014-1025. [CrossRef] [PubMed]

39. Goertz, D.E.; Wright, C.; Hynynen, K. Contrast agent kinetics in the rabbit brain during exposure to therapeutic ultrasound. Ultrasound Med. Biol. 2010, 36, 916-924. [CrossRef] [PubMed]

40. Pouliopoulos, A.N.; Bonaccorsi, S.; Choi, J.J. Exploiting flow to control the in vitro spatiotemporal distribution of microbubble-seeded acoustic cavitation activity in ultrasound therapy. Phys. Med. Biol. 2014, 59, 6941-6957. [CrossRef] [PubMed]

41. Pouliopoulos, A.N.; Li, C.; Tinguely, M.; Garbin, V.; Tang, M.X.; Choi, J.J. Rapid short-pulse sequences enhance the spatiotemporal uniformity of acoustically driven microbubble activity during flow conditions. J. Acoust. Soc. Am. 2016, 140, 2469-2480. [CrossRef]

42. Wu, F.; Chen, W.Z.; Bai, J.; Zou, J.Z.; Wang, Z.L.; Zhu, H.; Wang, Z.B. Pathological changes in human malignant carcinoma treated with high-intensity focused ultrasound. Ultrasound Med. Biol. 2001, 27, 1099-1106. [CrossRef]

43. Chen, W.; Zhu, H.; Zhang, L.; Li, K.; Su, H.; Jin, C.; Zhou, K.; Bai, J.; Wu, F.; Wang, Z. Primary bone malignancy: Effective treatment with high-intensity focused ultrasound ablation. Radiology 2010, 255, 967-978. [CrossRef] [PubMed]

44. Napoli, A.; Anzidei, M.; Marincola, B.C.; Brachetti, G.; Noce, V.; Boni, F.; Bertaccini, L.; Passariello, R.; Catalano, C. MR imaging-guided focused ultrasound for treatment of bone metastasis. Radiographics 2013, 33, 1555-1568. [CrossRef] [PubMed]

45. Shamout, F.E.; Pouliopoulos, A.N.; Lee, P.; Bonaccorsi, S.; Towhidi, L.; Krams, R.; Choi, J.J. Enhancement of non-invasive trans-membrane drug delivery using ultrasound and microbubbles during physiologically relevant flow. Ultrasound Med. Biol. 2015, 41, 2435-2448. [CrossRef]

(C) 2019 by the authors. Licensee MDPI, Basel, Switzerland. This article is an open access article distributed under the terms and conditions of the Creative Commons Attribution (CC BY) license (http://creativecommons.org/licenses/by/4.0/). 